\title{
Convergent regulatory evolution and loss of flight in paleognathous birds
}

\section{Citation}

Sackton, Timothy B., Phil Grayson, Alison Cloutier, Zhirui Hu, Jun S. Liu, Nicole E. Wheeler, Paul P. Gardner, et al. 2019. Convergent Regulatory Evolution and Loss of Flight in Paleognathous Birds. Science 364 (6435): 74-78.

\section{Permanent link}

http://nrs.harvard.edu/urn-3:HUL.InstRepos:39865637

\section{Terms of Use}

This article was downloaded from Harvard University's DASH repository, and is made available under the terms and conditions applicable to Open Access Policy Articles, as set forth at http:// nrs.harvard.edu/urn-3:HUL.InstRepos:dash.current.terms-of-use\#OAP

\section{Share Your Story}

The Harvard community has made this article openly available.

Please share how this access benefits you. Submit a story.

Accessibility 


\section{Convergent regulatory evolution and loss of flight in palaeognathous birds}

Timothy B. Sackton* (1,2), Phil Grayson (2,3), Alison Cloutier (2,3), Zhirui Hu (4), Jun S. Liu (4), Nicole E. Wheeler (5,6), Paul P. Gardner (5,7), Julia A. Clarke (8), Allan J. Baker (9,10), Michele Clamp (1), Scott V. Edwards* $(2,3)$

Affiliations:

1) Informatics Group, Harvard University, Cambridge, USA

2) Department of Organismic and Evolutionary Biology, Harvard University, Cambridge, USA

3) Museum of Comparative Zoology, Harvard University, Cambridge, USA

4) Department of Statistics, Harvard University, Cambridge, USA

5) School of Biological Sciences, University of Canterbury, New Zealand

6) Wellcome Sanger Institute, Wellcome Genome Campus, Cambridge, UK

7) Department of Biochemistry, University of Otago, New Zealand

8) Jackson School of Geosciences, The University of Texas at Austin, Austin, USA

9) Department of Natural History, Royal Ontario Museum, Toronto, Canada

10) Department of Ecology and Evolutionary Biology, University of Toronto, Toronto, Canada

*correspondence to: TBS (tsackton@g.harvard.edu) or SVE (sedwards@,fas.harvard.edu) 
Whether convergent phenotypic evolution is driven by convergent molecular changes, in proteins or regulatory regions, are core questions in evolutionary biology. Here we combine phylogenomic, developmental and epigenomic analysis of eleven new genomes of palaeognathous birds, including an extinct moa, to show that convergent evolution of regulatory regions, more so than protein-coding genes, is prevalent among developmental pathways associated with independent losses of flight. A novel Bayesian analysis of 280,000 conserved noncoding elements, 60,665 of which are corroborated as enhancers by open chromatin states during development, identifies 2,355 independent accelerations along lineages of flightless palaeognaths, with functional consequences for driving gene expression in the developing forelimb. Our results suggest that the genomic landscape associated with morphological convergence in ratites has a substantial shared regulatory component.

Convergent evolution - the independent evolution of similar phenotypes in divergent taxa - produces some of the most striking examples of adaptation, but the molecular architecture of convergent traits is not well understood (1-4). In some cases, similar or identical mutations in independent lineages appear to be associated with convergent phenotypes $(5,6)$, whereas in other cases convergent phenotypes appear to arise by diverse molecular paths (7). Whereas studies of convergence are increasingly focused on detecting genome-wide patterns $(8-13)$, the relative contributions of regulatory regions and protein-coding genes to convergent phenotypes remains an open question.

A particularly notable example of a convergent trait involves loss of powered flight, which has occurred many times independently in the course of avian evolution. One of the most iconic groups of flightless birds is the ratites, consisting of extant ostrich, kiwi, rheas, cassowaries, and emu, and the extinct moa and elephant birds. All ratites show morphological similarities including forelimb reduction (ranging from moderate in ostrich and rheas to a complete absence in moa), reduced pectoral muscle mass associated with the absence of the sternal keel, and feather modifications, as well as generally larger body size (14-16). Historically, ratites were thought to be a monophyletic sister clade to the volant tinamous (17), but, despite remaining uncertainties in palaeognath relationships, recent molecular phylogenetic evidence strongly supports ratite paraphyly, implying as many as six independent losses of flight within this group based on phylogenetic relationships and biogeographic scenarios $(16,18-22)$. 
To study the genomic correlates of flight loss in ratites, we assembled and annotated 11 new palaeognath genomes (Table S1-3, Fig. S1; (23)), including 8 flightless ratites (greater rhea, lesser rhea, cassowary, emu, Okarito kiwi, great spotted kiwi, little spotted kiwi, and the extinct little bush moa $(19,24)$ ) and 3 tinamous (thicket tinamou, elegant crested tinamou, and Chilean tinamou), and analyzed them together with the published ostrich, white-throated tinamou (25), and North Island brown kiwi (26) genomes.

We complied a total data set of 41,184,181 bp of aligned DNA from 20,850 noncoding loci, including introns, ultraconserved elements (27) and conserved non-exonic elements (CNEEs; Table S4; $(23,28)$ ) to test the hypothesis of ratite paraphyly and to resolve the placement of rheas, which remains an outstanding question in palaeognath phylogenetics $(18,22$, 29). Consistent with recent molecular phylogenies (18-22, 29), we recover a basal divergence between the ostrich and the remaining palaeognaths, including the tinamous, which are therefore nested within a paraphyletic ratite clade (Fig. 1a). However, contrary to recent concatenation analyses (22), our coalescent analysis using MP-EST (30) consistently places the rheas as sister to the kiwi+emu+cassowary clade (Fig. S2-S3). Our species tree is further corroborated by 4,274 informative CR1 retroelement insertions, including 18 absent in ostrich but shared among all non-ostrich palaeognaths, including tinamous (31). Discrepancies with previous analyses are explained by the existence of an empirical anomaly zone (32) in palaeognaths, in which incomplete lineage sorting across short internal branches leading to the ancestor of rheas, kiwi and emu+cassowary results in a set of most common gene trees that differ from the species tree (Fig. 1b, Fig. S4-S5), compromising the concatenation approach. Our analysis and biogeographic considerations (20-22) imply from 3-6 losses of flight in the history of this group (Fig. 1a). The alternative - a single loss of flight at the base of the palaeognaths followed by a regain of flight in tinamous - appears implausible given evidence for repeated losses of flight across birds and the lack of any evidence for regains of flight after loss (Fig. S6).

We analyzed 7,654 high quality gene alignments across 44 species of birds, including 9 flightless ratites, 4 volant tinamous, and 21 neognaths to test for convergent evolution in protein coding genes among ratite lineages (23). We find no evidence for an excess of convergent amino acid substitutions among ratites (Table S5), and minimal evidence for ratite-specific convergent shifts in rates of amino acid divergence (Table S6, Fig. S7). However, we do find 238 genes exhibiting positive selection on a subset of codons specifically in ratites, including 63 that are 
selected in multiple species, and an additional 294 genes with positive selection predominately in ratites ( $>50 \%$ of selected branches are ratites).

The number of convergently selected genes in ratites is greater than expected under the assumption of independent selection in each lineage (permutation $\mathrm{P}<0.001$ ). However, the degree of convergence we observe declines substantially if we make the conservative assumption that only three independent losses of flight occurred (permutation $\mathrm{P}=0.0475$; Fig. S8); we find no cases of genes positively selected in three independent losses of flight under the conservative loss model (in which flight is lost independently only in moa, ostrich, and the rhea/emu/kiwi/cassowary clade; Table S7). We also find no evidence that the convergently selected genes are enriched for any GO or KEGG terms (Q value $>0.20$ for all categories after multiple test correction). The larger set of genes with selection specifically or predominately in ratites (regardless of degree of convergent selection) is enriched for terms involved in protein metabolic processes (Additional File S1), but not transcription factors or proteins with functions in development, suggesting that convergent protein-coding evolution in these species may be associated with other phenotypes, such as changes in feathers, body size, or other traits, but not morphological evolution of forelimbs and related skeletal phenotypes such as the loss of a keel.

We applied profile hidden Markov models to detect sequence changes that are underrepresented in homologs of a protein (33), similar to that used to identify putative functionaltering mutations in protein-coding genes associated with loss of flight in the Galapagos cormorant (34). This approach yielded a single gene, Neurofibromin 1 (NF1), showing evidence for convergent functional evolution in ratites (at a 5\% FDR), but we do not detect an excess of convergent function-alternating protein-coding substitutions compared to genome-wide distributions (Fig. S9). Little is known about NF1 function in chickens, but in mice it has been shown to be involved in skeletal (35) and skeletal muscle development (36).

Regulatory regions may be subject to less pleiotropic constraint than protein-coding genes $(5,37)$, and may be more likely to underlie convergent phenotypes than protein-coding genes if common pathways are involved. To identify candidate regulatory regions in palaeognath genomes, we used phylogenetic conservation (38) to identify 284,001 CNEEs, which are thought to have regulatory roles in birds (39) and other taxa (40-43). A novel Bayesian method (44) for detecting changes in conservation of these elements across the phylogeny identified 2,355 elements specifically accelerated in ratites, 256-630 of which have experienced multiple 
independent acceleration events depending on modeling assumptions (Fig. 2; Fig. S10, Table S8); these results are consistent across input datasets and parameter values (Fig. S11-12).

To determine if ratites experience more convergent acceleration in CNEEs than is typical for birds, we randomly sampled trios of non-sister neognaths and counted how many CNEEs were convergently accelerated in all three lineages using the posterior probabilities from an unrestricted Bayesian model (23). Among random trios of neognaths, we observe a mean of 1.99 convergently accelerated CNEEs (across 1,552 permutations). In contrast, we observe a mean of 45.9 convergently accelerated CNEEs among seven ratite trios (under the conservative model of three independent losses of flight in moa, ostrich, and the ancestor of rhea/kiwi/cassowary/emu). The mean number of convergently accelerated CNEEs we observe in ratites is thus unusually high compared to an empirical null distribution (permutation P-value $=0.0064$, Fig. 3a). Although incomplete lineage sorting can increase apparent substitution rates (45), we find no evidence that ratite-accelerated elements are associated with increased gene tree discordance (Fig. S13).

We looked for evidence of functional convergence by testing for spatial and pathway enrichment among both the 2,355 ratite-specific accelerated CNEEs and the subset that are convergently accelerated in multiple lineages. Convergently accelerated CNEEs are enriched near genes associated with Gene Ontology terms including sequence-specific DNA binding, limb morphogenesis, Wnt signaling, and regulation of epithelial cell proliferation (Fig. S14, Additional File S2). Both ratite-accelerated and convergently accelerated CNEEs are overrepresented near a variety of genes of relevance to morphology and development (Fig. 3b), are spatially clustered along the genome (Fig. 3c), and include at least one element (mCE1916069) overlapping a known limb enhancer in mammals. No single gene or accelerated element is expected to account for the full extent of morphological convergence among ratites; however, accelerated elements are enriched near key limb developmental genes including TBX5, DACH1, PAX9, and genes from the IRX family (Fig. 3b, 3c). TBX5 is essential for forelimb development in tetrapods (46) and pectoral fin development in fish (47), and has been previously implicated in morphological divergence in emus (15). IRX5 is critical for both size and anteroposterior patterning in the limbs (48). DACH1 has been shown to repress BMP, promote proximodistal patterning, and maintain the apical ectodermal ridge during limb development (49). Finally, PAX9-deficient mice have craniofacial and limb abnormalities, alongside a 
complete loss of teeth (50). Some genes enriched for ratite-accelerated elements are also reported near clusters of lineage-accelerated elements in mammals, including DACH1, NPAS3, and NF1B in humans (51), HoxD in bats (52), and NPAS3 in primates (53). Deviations from model expectations, such as base compositional shifts (which some accelerated elements exhibit and could be driven by gene conversion) and presence of simple sequence repeats (54), appear unlikely to explain this overlap (Fig. S15), suggesting that certain key developmental genes are reused repeatedly during morphological evolution across amniotes, or that a high density of nearby regulators predisposes certain genomic regions to repeated evolution.

Ratite accelerated elements are candidates for regulatory regions associated with the range of convergent phenotypes associated with loss of flight in ratites, although we expect this suite of traits to be highly polygenic. To functionally characterize the association between sequence conservation and regulatory activity in birds, we used ATAC-seq to identify genomic regions of open chromatin for 8 tissues during the course of chick development $(55,56)$. Consistent with recent work (39), CNEEs are enriched under ATAC-seq peaks (Fig. 4a), supporting the regulatory function of CNEEs in this and other datasets. To test whether ratitespecific sequence acceleration results in functional differentiation of enhancer activity in vivo, we screened a set of candidates for enhancer activity in developing chicken forelimbs. We focused on putative forelimb enhancers identified by examining the intersection of consistent forelimb ATAC-seq peaks, convergent ratite-accelerated elements, and previously published embryonic chicken ChIP-seq peaks, resulting in 54 candidate enhancers (Fig. 4b, Additional File S3). Using an electroporated $\beta$-actin/GFP enhancer construct assay, we identified a promising chicken region from among these candidates, consisting of the ATAC-seq peak containing the with convergent ratite-accelerated element mCE967994, which produced consistent strong enhancer activity in early chicken forelimbs (Fig. 4c-e). This element is located in the intron of the TEADl gene, which has been implicated in cell proliferation, cell survival, mesoderm patterning, and the epithelial-mesenchymal transition (57-59), and contains binding sites for TEF-1/TEAD1, suggesting it may play a role in TEAD1 autoregulation. We tested for enhancer activity of the homologous genomic region in the volant elegant crested tinamou, in which mCE967994 is identified as conserved in our Bayesian analysis, and the flightless greater rhea, in which mCE967994 is accelerated. We find that the tinamou version of this enhancer consistently drives GFP expression ( $\mathrm{N}=9$ of 10 embryos), but the rhea version does not (Fig. 4e; 
$\mathrm{N}=10$ of 13 embryos). Thus, accelerated sequence evolution of this element in rheas appears associated with functional divergence of regulatory activity. Although we cannot rule out the possibility that compensatory trans-regulatory evolution in rheas maintains function of this and other accelerated elements in their natural context, this assay demonstrates that our unbiased comparative genomic screen readily identifies cis-regulatory elements with functionally divergent cis-regulatory activity in vivo.

Understanding the relative roles of regulatory and protein-coding change in phenotypic evolution, as well as genomic mechanisms underlying convergent phenotypes, are longstanding questions in evolutionary biology (60). Our unbiased statistical and functional screens, which emphasized genomic changes occurring in parallel on multiple lineages of ratites, suggest that convergent morphological evolution and loss of flight in ratites is associated more strongly with regulatory evolution in noncoding DNA than with evolutionary changes in protein-coding genes, and contrast with previous work, which emphasized protein-coding correlates of flightlessness in birds. Although our results do not rule out a role for lineage-specific genomic drivers of flightlessness, they provide a template for future genome-wide studies of loss of flight and other convergent phenotypes across the Tree of Life. 


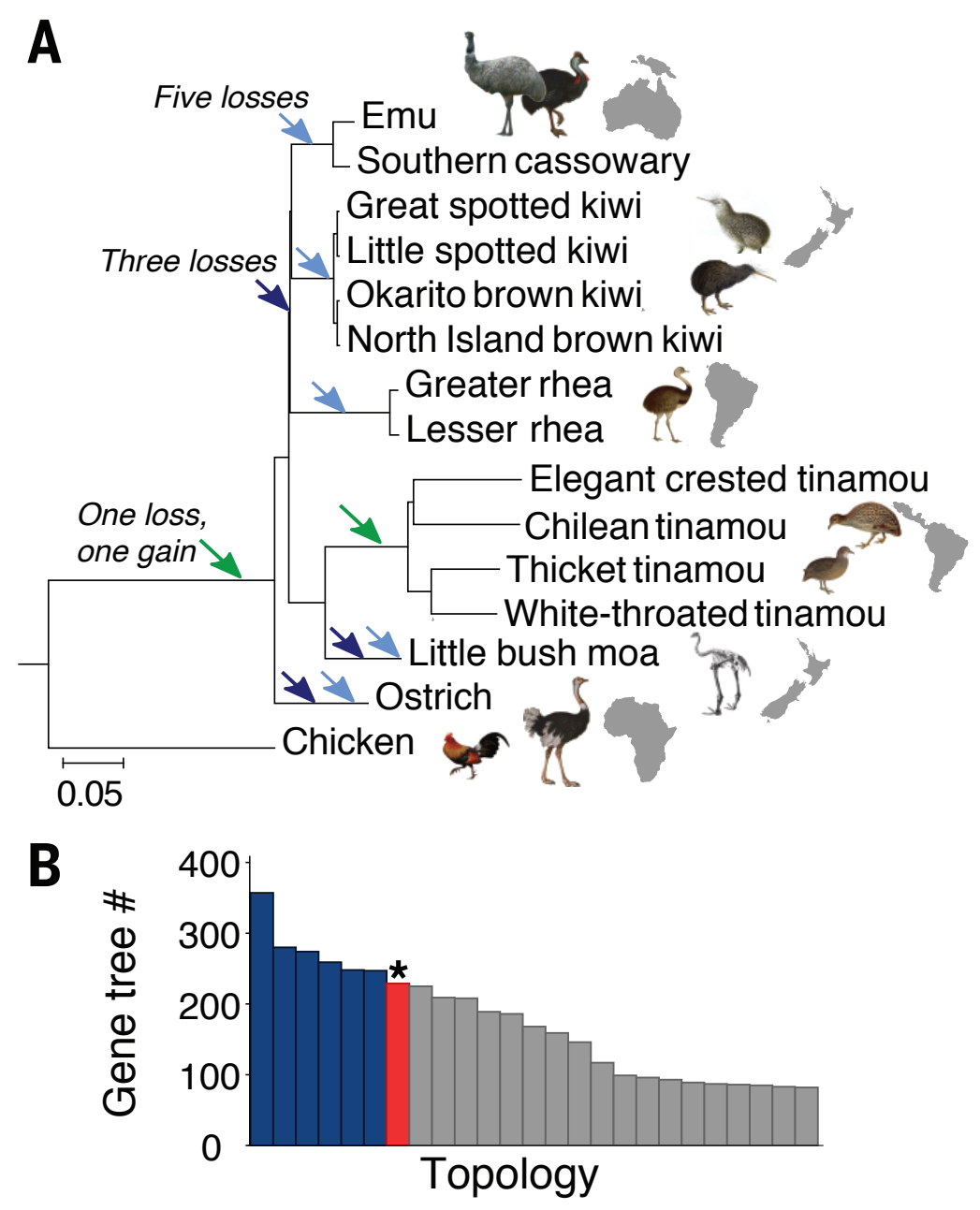

Figure 1: Genome-wide data places rheas as sister to a kiwi/cassowary-emu clade. A) MPEST species tree topology, with 100\% bootstrap support throughout (not shown). Branch lengths in units of substitutions per site were estimated with ExaML using a fully partitioned alignment of 20,850 noncoding loci constrained to the MP-EST topology. Ratite paraphyly is consistent with either a single loss of flight in the palaeognath ancestor followed by regain in tinamous (green arrows) or a minimum of three independent losses of flight in the ratites (dark blue arrows), with at least five losses suggested by a proposed sister group relationship between elephant birds and kiwi (light blue arrows; elephant bird branch not shown). B) Distribution of the 25 most common gene tree topologies, showing that the species tree topology (in red and marked with a star symbol) is not the most common gene tree topology. 


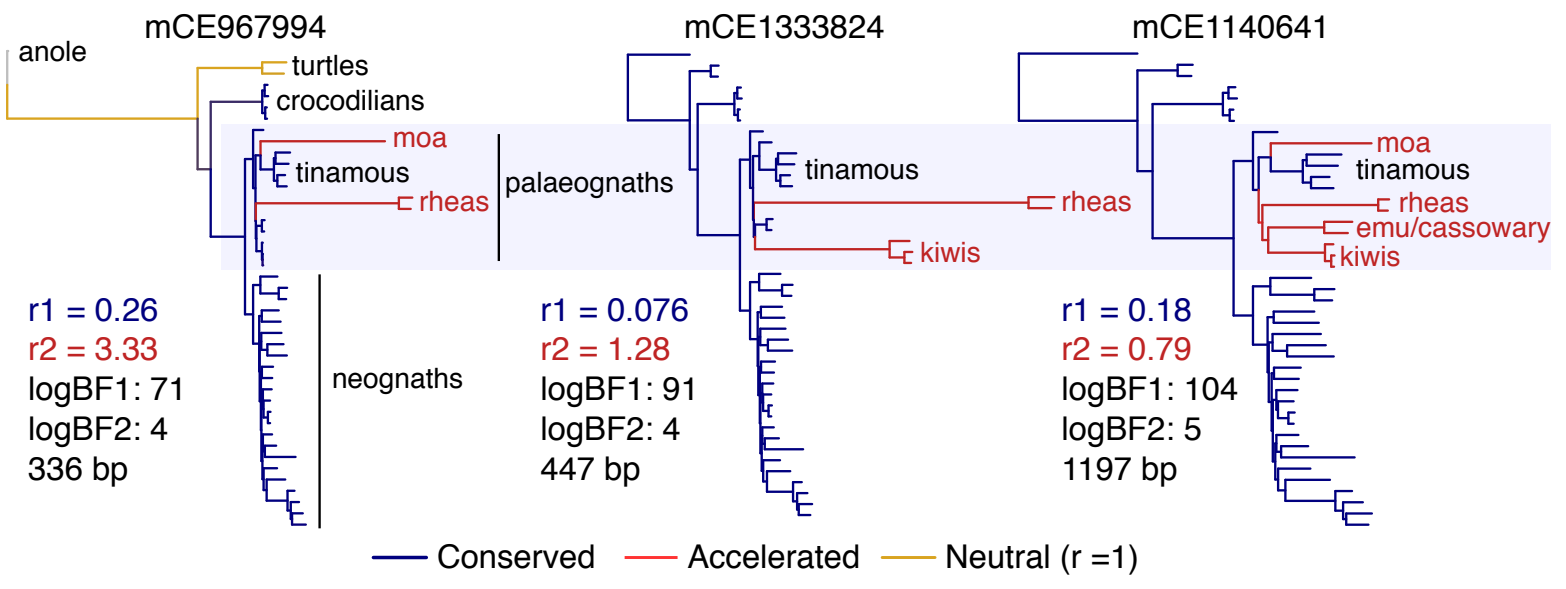

Figure 2. Novel Bayesian model identifies convergently accelerated CNEEs in ratites. Trees for three examples of convergent ratite accelerated elements (specific CNEE ids are listed above each tree). Major groups of birds and non-avian outgroups are indicated on the leftmost tree. Branch lengths are proportional to substitution rate relative to the neutral rate $(r=1)$ and branches are colored according to the maximum posterior conservation state. Bayes Factors (BFs) 1 and 2 (see Supplementary Material and ref. 44), the conserved $\left(r_{1}\right)$ and accelerated $\left(r_{2}\right)$ rates, and length of each element in base pairs are indicated. Gray branches indicate the element is missing or ambiguous for that taxon. 


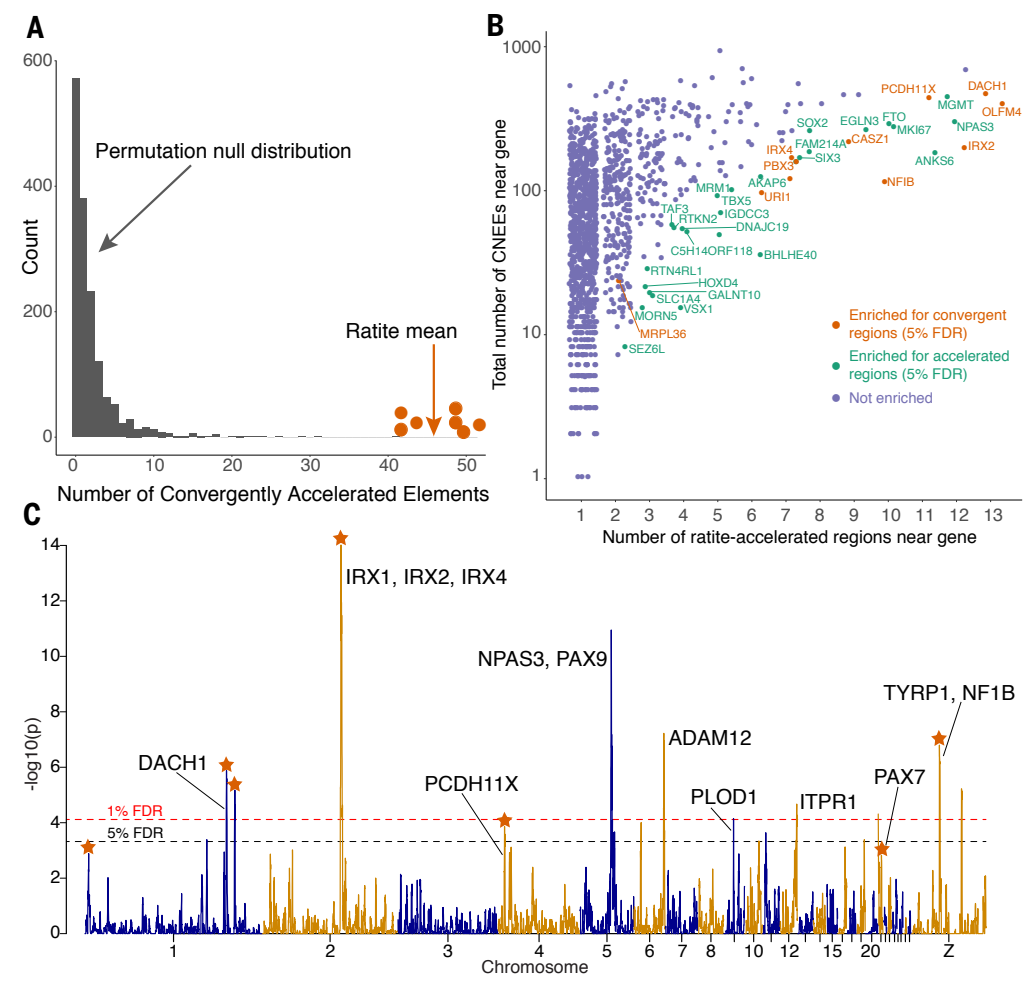

Figure 3. Ratites exhibit unusually high numbers of convergently accelerated CNEEs. A) Histogram of the null distribution of the observed number of convergently accelerated elements in random trios of neognaths (gray), with individual ratite trios (red points) and ratite mean (red arrow) indicated; ratites have significant excess of convergence (permutation P-value $=0.0064$ ). B) Genes with evidence for excess of nearby accelerated and convergently accelerated elements. Colored and labeled points are genes with excess convergent or accelerated elements based on a permutation test (5\% FDR). Only genes with at least one accelerated element are plotted; raw data underlying plot is provided as Additional File S4. C) Evidence for spatial clustering of accelerated elements across palaeognath genomes. The y-axis shows the negative $\log 10 \mathrm{p}$-value for a test of excess convergent ratite accelerated elements in $1 \mathrm{Mb}$ sliding windows (100kb slide), computed using a binomial test, where the probability of success is the total accelerated elements divided by the total number of elements, and the number of samples is the number of CNEEs in each window. Genes that may be of particular interest are noted; windows with an excess of convergently accelerated elements are indicated with a star. The reference coordinates are the chicken genome (galGal4); significant windows are provided as Additional File S5. 

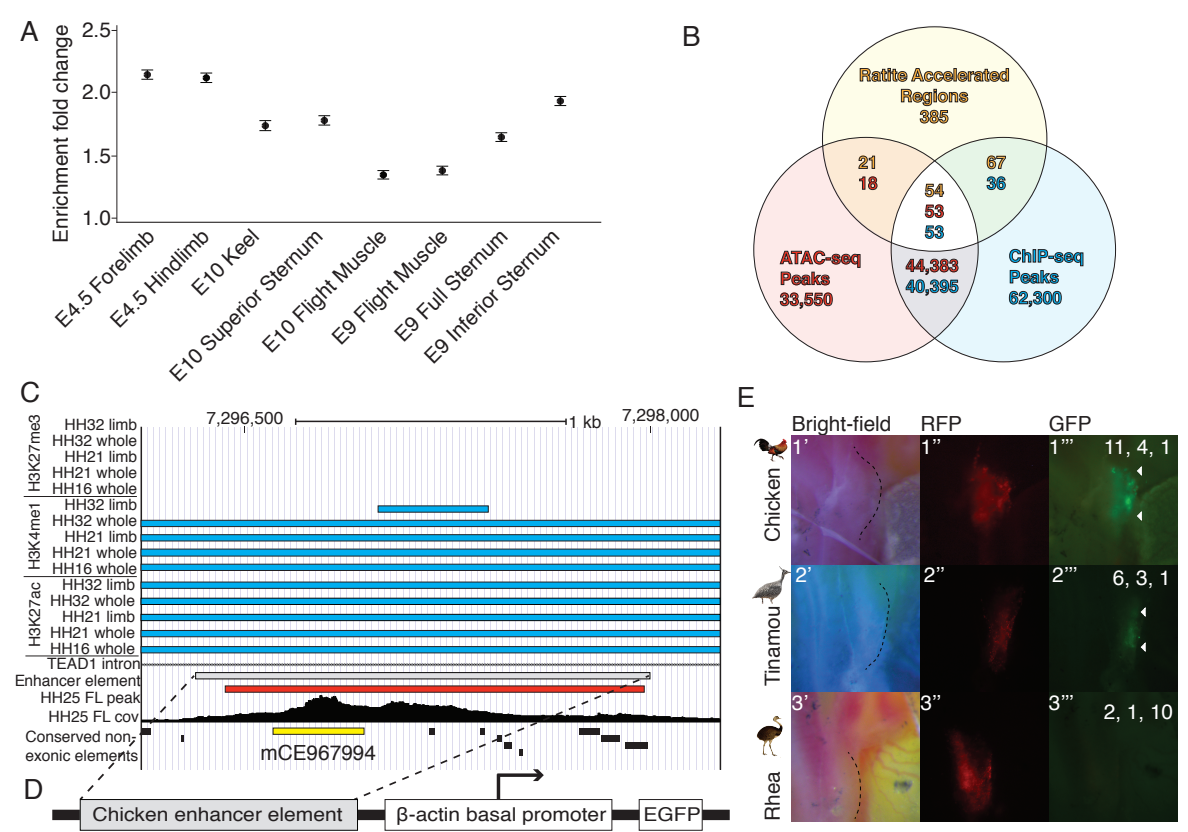

Figure 4. Ratite-specific convergent acceleration is associated with altered activity in a candidate enhancer. A) Conserved non-exonic elements are enriched under ATAC-seq peaks across all eight chicken tissues. Enrichment fold change (mean and 95\% CI), calculated against chicken genomic background using GAT (10,000 samples). B) Candidate forelimb ATAC-seq peaks for enhancer screen were identified by overlap with convergently accelerated elements and ChIP-seq peaks. C) Genomic region associated with strong enhancer activity in early chicken forelimbs. D) Schematic of $\beta$-actin/GFP vector used in enhancer screen. E) Results of enhancer screen of candidate ATAC-seq peak in chicken forelimb bud. Pictures are representative images of 10-16 replicates showing developing forelimb region 24 hours after electroporation (HH1920). Dashed line on the bright field image (left column) indicates the forelimb region. RFP expression (red, middle column) indicates the area electroporated. GFP expression (green, right column) is driven by enhancer activity of the candidate region. Number of replicates showing strong, partial, and no GFP expression are indicated in upper right corner of each image. e1) Chicken ATAC-seq peak drives consistent GFP expression throughout the developing forelimb (strong GFP 11/16, partial GFP 4/16, no GFP 1/16). e2) The homologous genomic region in the elegant crested tinamou also drives consistent GFP expression throughout the developing chicken forelimb (strong GFP 6/10, partial GFP 3/10, no GFP 1/10). e3) The homologous genomic region in the greater rhea fails to drive consistent GFP expression throughout the developing chicken forelimb (strong GFP 2/13, partial GFP 1/13, no GFP 10/13). 


\section{References Cited}

1. J. B. Losos, Evolution. 65, 1827-40 (2011).

2. A. Martin, V. Orgogozo, Evolution. 67, 1235-50 (2013).

3. D. L. Stern, Nat Rev Genet. 14, 751-64 (2013).

4. J. F. Storz, Nat Rev Genet. 17, 239-50 (2016).

5. Y. F. Chan et al., Science. 327, 302-305 (2010).

6. Y. Zhen, M. L. Aardema, E. M. Medina, M. Schumer, P. Andolfatto, Science. 337, 16341637 (2012).

7. K. L. Cooper et al., Nature. 511, 41-45 (2014).

8. S. Yeaman et al., Science. 353, 1431-1433 (2016).

9. R. Partha et al., Elife. 6 (2017), doi:10.7554/eLife.25884.

10. S. Xu et al., Mol Biol Evol. 34, 1008-1015 (2017).

11. M. J. Berger, A. M. Wenger, H. Guturu, G. Bejerano, Nucleic Acids Res. 46, 9299-9308 (2018).

12. W. K. Meyer et al., Science. 361, 591-594 (2018).

13. V. Sharma et al., Nat. Commun. 9, 1215 (2018).

14. P. W. Houde, Nature. 324, 563-565 (1986).

15. S. R. B. Bickley, M. P. O. Logan, Proc. Natl. Acad. Sci. 111, 17917-17922 (2014).

16. S. J. Nesbitt, J. A. Clarke, Bull. Am. Mus. Nat. Hist., 1-91 (2016).

17. J. Cracraft, Ibis. 116, 494-521 (1974).

18. J. Harshman et al., Proc. Natl. Acad. Sci. U. S. A. 105, 13462-13467 (2008).

19. A. J. Baker, O. Haddrath, J. D. McPherson, A. Cloutier, Mol. Biol. Evol. 31, 1686-1696 (2014).

20. K. J. Mitchell et al., Science. 344, 898-900 (2014).

21. A. Grealy et al., Mol. Phylogenet. Evol. 109, 151-163 (2017).

22. T. Yonezawa et al., Curr Biol. 27, 68-77 (2017). 
23. Materials and methods are available as supplementary materials at the Science website.

24. A. Cloutier, et al bioRxiv (2018).

25. E. D. Jarvis et al., Science. 346, 1320-1331 (2014).

26. D. Le Duc et al., Genome Biol. 16, 147 (2015).

27. J. E. McCormack et al., Genome Res. 22, 746-754 (2012).

28. S. V. Edwards, A. Cloutier, A. J. Baker, Syst. Biol. 66, 1028-1044 (2017).

29. M. J. Phillips, G. C. Gibb, E. A. Crimp, D. Penny, Syst Biol. 59, 90-107 (2010).

30. L. Liu, L. Yu, S. V. Edwards, BMC Evol. Biol. 10, 302 (2010).

31. A. Cloutier et al., bioRxiv (2018), doi:10.1101/262949.

32. J. H. Degnan, N. A. Rosenberg, Public Libr. Sci. Genet. 2, 762-768 (2006).

33. N. E. Wheeler, L. Barquist, R. A. Kingsley, P. P. Gardner, Bioinformatics. 32, 3566-3574 (2016).

34. A. Burga et al., Science. 356 (2017), doi:10.1126/science.aal3345.

35. M. Kolanczyk et al., Hum Mol Genet. 16, 874-86 (2007).

36. N. Kossler et al., Hum. Mol. Genet. 20, 2697-2709 (2011).

37. S. B. Carroll, B. Prud'homme, N. Gompel, Sci Am. 298, 60-7 (2008).

38. A. Siepel et al., Genome Res. 15, 1034-50 (2005).

39. R. Seki et al., Nat Commun. 8, 14229 (2017).

40. L. A. Pennacchio et al., Nature. 444, 499-502 (2006).

41. A. Visel et al., Nat Genet. 40, 158-60 (2008).

42. J. A. Capra, G. D. Erwin, G. McKinsey, J. L. R. Rubenstein, K. S. Pollard, Philos. Trans. R. Soc. B Biol. Sci. 368, 20130025-20130025 (2013).

43. D. E. Dickel et al., Cell. 172, 491-499.e15 (2018).

44. Z. Hu, T. B. Sackton, S. V. Edwards, J. S. Liu, Mol. Biol. Evol. in press (2019)

45. F. K. Mendes, M. W. Hahn, Syst Biol. 65, 711-21 (2016).

46. C. Rallis, Development. 130, 2741-2751 (2003). 
47. D. Ahn, M. J. Kourakis, L. A. Rohde, L. M. Silver, R. K. Ho, Nature. 417, 754-758 (2002).

48. D. Li et al., Dev. Cell. 29, 233-240 (2014).

49. Y. Kida, Development. 131, 4179-4187 (2004).

50. H. Peters, A. Neubüser, K. Kratochwil, R. Balling, Genes Dev. 12, 2735-2747 (1998).

51. K. S. Pollard et al., PLOS Genet. 2, e168 (2006).

52. B. M. Booker et al., "Bat Accelerated Regions Identify a Bat Forelimb Specific Enhancer in the HoxD Locus" (2015).

53. D. Kostka, A. K. Holloway, K. S. Pollard, Mol. Biol. Evol. 35, 2034-2045 (2018).

54. K. T. Xie et al., Science. 363, 81-84 (2019).

55. J. D. Buenrostro, P. G. Giresi, L. C. Zaba, H. Y. Chang, W. J. Greenleaf, Nat Methods. 10, 1213-8 (2013).

56. A. R. Gehrke et al., Proc Natl Acad Sci U A. 112, 803-8 (2015).

57. H. Zhang et al., J. Biol. Chem. 284, 13355-13362 (2009).

58. B. Zhao et al., Genes Dev. 22, 1962-1971 (2008).

59. A. Sawada et al., Mol. Cell. Biol. 28, 3177 (2008).

60. Lamichhaney, S. et al., Philos. Trans. R. Soc. B Biol. Sci. in press (2019).

\section{Additional References Cited}

61. P. Grayson, S. Y. W. Sin, T. B. Sackton, S. V. Edwards, in Avian and Reptilian Developmental Biology (Humana Press, New York, NY, 2017;

https://link.springer.com/protocol/10.1007/978-1-4939-7216-6_2), Methods in Molecular Biology, pp. 11-46.

62. J. Butler et al., Genome Res. 18, 810-820 (2008).

63. A. M. Bolger, M. Lohse, B. Usadel, Bioinforma. Oxf. Engl. 30, 2114-2120 (2014).

64. F. A. Simão, R. M. Waterhouse, P. Ioannidis, E. V. Kriventseva, E. M. Zdobnov, Bioinforma. Oxf. Engl. 31, 3210-3212 (2015).

65. E. Hagelberg, in Ancient DNA (Springer, New York, NY, 1994;

https://link.springer.com/chapter/10.1007/978-1-4612-4318-2_13), pp. 195-204.

66. A. Cooper, H. N. Poinar, Science. 289, 1139-1139 (2000). 
67. M. Knapp, A. C. Clarke, K. A. Horsburgh, E. A. Matisoo-Smith, Ann. Anat. Anat. Anz. Off. Organ Anat. Ges. 194, 3-6 (2012).

68. H. Li et al., Bioinformatics. 25, 2078-2079 (2009).

69. B. Langmead, S. L. Salzberg, Nat. Methods. 9, 357-359 (2012).

70. H. Jónsson, A. Ginolhac, M. Schubert, P. L. F. Johnson, L. Orlando, Bioinformatics. 29, 1682-1684 (2013).

71. J. Zhang, K. Kobert, T. Flouri, A. Stamatakis, Bioinformatics. 30, 614 (2014).

72. C. Holt, M. Yandell, BMC Bioinformatics. 12, 491 (2011).

73. B. Vicoso, V. B. Kaiser, D. Bachtrog, Proc. Natl. Acad. Sci. 110, 6453-6458 (2013).

74. B. J. Haas et al., Nat. Protoc. 8, 1494-1512 (2013).

75. D. Kim et al., Genome Biol. 14, R36 (2013).

76. S. R. Eddy, PLoS Comput. Biol. 7, e1002195 (2011).

77. S. Powell et al., Nucleic Acids Res. 40, D284-289 (2012).

78. A. M. Altenhoff, M. Gil, G. H. Gonnet, C. Dessimoz, PLoS ONE. 8, e53786 (2013).

79. K. Katoh, D. M. Standley, Mol. Biol. Evol. 30, 772-780 (2013).

80. C. Trapnell et al., Nat. Biotechnol. 28, 511-515 (2010).

81. A. Löytynoja, Methods Mol. Biol. Clifton NJ. 1079, 155-170 (2014).

82. A. Stamatakis, Bioinformatics. 30, 1312-1313 (2014).

83. B. Paten et al., Genome Res. 21, 1512-1528 (2011).

84. R. E. Green et al., Science. 346, 1254449 (2014).

85. M. J. Hubisz, K. S. Pollard, A. Siepel, Brief. Bioinform. 12, 41-51 (2011).

86. A. R. Quinlan, I. M. Hall, Bioinformatics. 26, 841-842 (2010).

87. G. Hickey, B. Paten, D. Earl, D. Zerbino, D. Haussler, Bioinforma. Oxf. Engl. 29, 13411342 (2013).

88. S. Capella-Gutiérrez, J. M. Silla-Martínez, T. Gabaldón, Bioinforma. Oxf. Engl. 25, 19721973 (2009).

89. A. M. Kozlov, A. J. Aberer, A. Stamatakis, Bioinforma. Oxf. Engl. 31, 2577-2579 (2015). 
90. J. Huerta-Cepas, F. Serra, P. Bork, Mol. Biol. Evol. 33, 1635-1638 (2016).

91. D. L. Swofford, PAUP*: Phylogenetic Analysis Using Parsimony (and other methods) 4.0.b5 (2001).

92. Maddison, W. P., Maddison, D. R., Mesquite: a modular system for evolutionary analysis (2018; http://www.mesquiteproject.org).

93. J. M. Beaulieu, B. C. O’Meara, Syst. Biol. 65, 583-601 (2016).

94. E. Paradis, K. Schliep, Bioinforma. Oxf. Engl. (2018), doi:10.1093/bioinformatics/bty633.

95. Z. Yang, Mol. Biol. Evol. 24, 1586-1591 (2007).

96. S. L. K. Pond, S. D. W. Frost, S. V. Muse, Bioinformatics. 21, 676-679 (2005).

97. S. L. Kosakovsky Pond et al., Mol. Biol. Evol. 28, 3033-3043 (2011).

98. M. D. Smith et al., Mol. Biol. Evol. 32, 1342-1353 (2015).

99. J. O. Wertheim, B. Murrell, M. D. Smith, S. L. Kosakovsky Pond, K. Scheffler, Mol Biol Evol. 32, 820-32 (2015).

100. A. Kowalczyk et al., bioRxiv, 451138 (2018).

101. R. Partha, A. Kowalczyk, N. L. Clark, M. Chikina, bioRxiv, 457309 (2018).

102. M. Chikina, J. D. Robinson, N. L. Clark, Mol Biol Evol. 33, 2182-92 (2016).

103. G. Yu, L.-G. Wang, Y. Han, Q.-Y. He, OMICS J. Integr. Biol. 16, 284-287 (2012).

104. D. Orme, 36 (2018).

105. K. S. Pollard, M. J. Hubisz, K. R. Rosenbloom, A. Siepel, Genome Res. 20, 110-121 (2010).

106. A. Heger, C. Webber, M. Goodson, C. P. Ponting, G. Lunter, Bioinforma. Oxf. Engl. 29, 2046-2048 (2013).

107. E. Calo, J. Wysocka, Mol. Cell. 49, 825-837 (2013).

108. H. Ochi et al., Nat. Commun. 3, 848 (2012).

109. K. Onimaru et al., eLife. 4, doi:10.7554/eLife.07048.

110. D. G. Gibson et al., Nat. Methods. 6, 343-345 (2009).

111. T. Suzuki, T. Ogura, Dev. Growth Differ. 50, 459-465 (2008). 
112. F. Supek, M. Bošnjak, N. Škunca, T. Šmuc, PLOS ONE. 6, e21800 (2011).

113. G. Zhang et al., Science. 346, 1311-1320 (2014).

\section{Acknowledgements}

We thank Carol Ritland, Myles Lamont, Kevin Kerr, Graham Crawshaw, Dan Janes, Rob Fleischer, and Jeremiah Trimble for assistance with sample acquisition. Terence Capellini, Clifford Tabin, John Young, Katie Pollard, Nicolas Lonfat, and Mariel Young provided advice, assistance, training, and equipment that enabled the developmental and genomic work reported here. Benedict Paten and Joel Armstrong provided assistance with progressiveCactus. Aaron Kitzmiller and James Cuff provided advice and support on computational and software issues. We gratefully acknowledge the Ngai Tahu and Te Ati Awa peoples, who permitted genetic analyses of kiwi blood samples obtained from their lands. We would like to acknowledge that this work was largely conducted on the traditional territory of the Wampanoag peoples. The computations in this paper were run on the Odyssey cluster supported by the FAS Division of Science, Research Computing Group at Harvard University, the Sanger Institute Computing Cluster, and the Abacus Computing Cluster at Canterbury University. Funding: This project was supported by NSF grant DEB-1355343/EAR-1355292 to SVE, AJB, JAC, and MC. PG was supported by an NSERC PGSD-3 grant. Publication costs were covered in part by a grant from the Wetmore Colles Fund of the Museum of Comparative Zoology. Author contributions: SVE, AJB, and JAC conceived this project with help from MC. SVE provided overall project leadership. AJB, AC, and SVE obtained samples. PG and AC prepared DNA, RNA, and sequencing libraries. TBS, SVE, and MC designed analysis strategies with assistance from all authors. TBS provided supervision for all bioinformatics, assembled and annotated all genomes, made the whole genome alignment, identified conserved elements, identified homologous proteins, and led the analysis of protein-coding evolution, non-coding evolution, and convergence. AC compiled protein, $\mathrm{CNEE}, \mathrm{UCE}$, and intron alignments and led the phylogenetics analysis. PG prepared samples for ATAC-seq, analyzed ATAC-seq data, conducted in silicio analyses on candidate accelerated elements, and carried out all developmental work. NEW and PPG developed and applied the DeltaBS method to detect functional divergence. ZH and JSL developed and applied the phyloAcc method. TBS wrote the manuscript together with AC, PG, and SVE. All authors edited the manuscript and approved the 
final draft. Competing interests: All authors declare that they have no competing interests. Data and materials availability: All sequencing data generated in this project is available at NCBI under BioProjects PRJNA433110 (extant genomes), PRJNA433423 (moa genome), PRJNA433154 (ATAC-seq), and PRJNA433114 (RNA-seq). In addition, processed data is available at Dryad and a UCSC track hub is publicly available (for viewing in the UCSC genome browser) at: https://ifx.rc.fas.harvard.edu/pub/ratiteHub8/hub.txt. Code availability: All scripts used for data processing and analysis are available on Github at https://github.com/tsackton/ratite-genomics. Further details are provided in the supplemental methods. 